\title{
Sizing and tailoring the Dacron graft for reimplantation of the aortic valve
}

Tirone E. David, MD

See related article on page 601 .

From the Department of Surgery, University of Toronto, Toronto, Ontario, Canada.

Received for publication March 16, 2005; accepted for publication April 1, 2005.

Address for reprints: Tirone E. David, MD, Department of Surgery, University of Toronto, 200 Elizabeth St, 13EN219, Toronto, Ontario M5G 2C4, Canada (E-mail: tirone.david@uhn.on.ca).

J Thorac Cardiovasc Surg 2005;130:243-4

$0022-5223 / \$ 30.00$

Copyright $\left({ }^{\circ} 2005\right.$ by The American Association for Thoracic Surgery

doi:10.1016/j.jtcvs.2005.04.002
I $\mathrm{n}$ this issue of the Journal, Thomas Gleason ${ }^{1}$ describes a new formulation to determine the diameter of the graft to be used for reimplantation of the aortic valve with creation of neoaortic sinuses. All his geometric assumptions are based on anatomic measurements of the normal aortic root. This is a problem because the anatomic components of the aortic valve in patients with aortic root aneurysm are almost invariably abnormal. The aortic annulus in these patients is often enlarged, and the height of the subcommissural triangles tends to be shorter than normal beneath the commissures of the noncoronary cusp. Those two triangles are more obtuse than normal. The aortic cusps are larger than usual and, more important, the free margins of one or more cusps are almost always elongated. The sinuses of Valsalva are aneurysmal, and the sinotubular junction is dilated. The more severe the aortic valve insufficiency, the more abnormal the components of the aortic root are, particularly the aortic cusps. Moreover, even in patients without aortic insufficiency, the anatomic components are abnormal. What makes aortic valve-sparing operations difficult is our inability to identify the abnormalities of the aortic root that need correction. The size and shape of the graft used for correction might be of secondary importance. Obviously, one cannot use one or two sizes of grafts for all patients, although that is what some surgeons now do by using commercially available grafts with already built sinuses, and they claim that the results are excellent. The diameter of the graft is crucial when the reimplantation technique is used because the aortic annulus and cusps are placed in the inside of the graft, and it has to be large enough to allow them to function normally. Although the study by Kunzelman and colleagues ${ }^{2}$ showed poor correlation between the height of the cusps and the diameters of the aortic annulus and sinotubular junction in the normal aortic root, I believe that during aortic valve-sparing operations, the height of the cusps is probably the most important measurement to estimate the diameter of the aortic annulus and sinotubular junction because it cannot be modified. Thus in our original description of aortic valve operations, ${ }^{3}$ the assumption that the radius of the reconstructed aortic root should be approximately two thirds of the average height of the cusps is not a bad one given the crescent shape of the aortic cusps and the fact that part of their surface is used for coaptation. We estimate the diameter of the graft by adding approximately $6 \mathrm{~mm}$ to the two thirds of the average height of the aortic cusps because the graft is placed on the outside of the aortic annulus. If a curvilinear shape beneath the central portion of the cusps is desirable, one can pick a graft of one size larger and place pleats in one of its ends in the areas corresponding to the nadir of the aortic annulus. However, when a straight tube is secured to the aortic annulus along a single horizontal plane, as we described originally, ${ }^{3}$ this curvilinear shape occurs because the sutures are tied on the outside of the graft, even if no pleats are used in the graft. We believe that it is extremely important to suture the graft below the level of the aortic annulus along a single horizontal plane in the fibrous components of the left ventricular outflow tract because that is the area the annulus dilates. Most grafts we use range from 30 to $36 \mathrm{~mm}$ in diameter.

Resuspension of the three commissures of the valve into the graft is also important for proper alignment and coaptation of the cusps. The three commissures are usually of different heights, and the one in between the right and left cusps is shorter. We try to place the two commissures of the noncoronary cusp at the same level in the graft and the one in between the left and right cusps at a slightly lower 
level. The remnants of the aortic sinuses and aortic annulus must be firmly sutured to the graft along a scalloped shape. Thus this second suture line remodels the aortic annulus and guarantees hemostasis.

The three cusps are then examined for the level of coaptation. Often, the free margin of one is larger than those of the others and requires reduction, which is done by plication of its central portion along the nodule of Aranti. If the free margin is stretched and thinned, we reinforce it with a double layer of 6-0 expanded polytetrafluoroethylene suture from commissure to commissure. Because it has been shown that creation of sinuses of Valsalva in the graft reduces the velocity of closure of the aortic cusps, we believe that the graft in between two commissures at the level of the sinotubular junction should be tailored slightly to create a spherical sinus. There is no study that has examined the relationship between the sphericity of the aortic sinuses and the velocity of closure of the cusps after aortic valve-sparing operations. Thus, the degree of curvature created by the pleats at the level of the sinotubular junction is largely empiric.

I do not agree with Gleason's modification of the annular suture line. The coronet-shaped subannular suture line described in this article and illustrated in Figure 2 from the article does not restore normal geometry to the subcommissural triangles. If aortic annuloplasty is not an important part of aortic valve-sparing operations, then the remodeling procedure is simpler and creates neoaortic sinuses better than the technique of reimplantation of the aortic valve. However, the main reason we developed the reimplantation techniques was to address the issue of aortic annulus dilation and distortion. As mentioned above, the aortic annulus of patients with aortic root aneurysms is dilated, and the heights of subcommissural triangles of the noncoronary cusp are shortened. Only reimplantation of the annulus and its subcommissural fibrous tissues into a cylinder can restore its 3-dimensional shape. After the aortic valve has been placed inside the graft, all three cusps must coapt at the same level, and this level should be at least 5 or $6 \mathrm{~mm}$ above the aortic annulus. Often we have to shorten the free margin of at least one cusp after reimplantation of the aortic valve to allow all three cusps to coapt at the same level.

Having said what I think and do, I have to commend Dr Gleason for attempting to add more science to make aortic valve reimplantation more reproducible and being successful in applying clinically the modification he described.

\section{References}

1. Gleason T. New graft formulation and modification of the David reimplantation technique. J Thorac Cardiovasc Surg. 2005;130:601-3.

2. Kunzelman KS, Grande KJ, David TE, Cochran RP, Verrier ED. Aortic root and valve relationships impact on surgical repair. J Thorac Cardiovasc Surg. 1994;107:162-70.

3. David TE, Feindel CM. An aortic valve-sparing operation for patients with aortic incompetence and aneurysm of the ascending aorta. J Thorac Cardiovasc Surg. 1992;103:617-22. 